\title{
Gravitational perturbations of Schwarzschild spacetime at null infinity and the hyperboloidal initial value problem
}

\author{
Anıl Zenginoğlu ${ }^{1,2,3}$, Darío Núñez ${ }^{3,4}$ and Sascha Husa ${ }^{3,5}$ \\ ${ }^{1}$ Department of Physics, University of Maryland, College Park, MD 20742, USA \\ 2 Center for Scientific Computation and Mathematical Modeling, University of Maryland, \\ College Park, MD 20742, USA \\ ${ }^{3}$ Max-Planck-Institut für Gravitationsphysik, Albert-Einstein-Institut, 14476 Golm, Germany \\ ${ }^{4}$ Instituto de Ciencias Nucleares, Universidad Nacional Autónoma de México, A.P. 70-543, \\ México D.F. 04510, México \\ ${ }^{5}$ Departament de Física, Universitat de les Illes Balears, Cra. Valldemossa Km. 7.5, E-07122 \\ Palma de Mallorca, Spain
}

Received 10 October 2008, in final form 21 November 2008

Published 13 January 2009

Online at stacks.iop.org/CQG/26/035009

\begin{abstract}
We study gravitational perturbations of Schwarzschild spacetime by solving a hyperboloidal initial value problem for the Bardeen-Press equation. Compactification along hyperboloidal surfaces in a scri-fixing gauge allows us to have access to the gravitational waveform at null infinity in a general setup. We argue that this hyperboloidal approach leads to a more accurate and efficient calculation of the radiation signal than the common approach where a timelike outer boundary is introduced. The method can be generalized to study perturbations of Kerr spacetime using the Teukolsky equation.
\end{abstract}

PACS numbers: $04.25 . \mathrm{Nx}, 04.25 . \mathrm{Dg}, 04.30 . \mathrm{Nk}, 04.20 . \mathrm{Ha}$

\section{Introduction}

In the last few years, numerical relativity has made tremendous progress in treating the inspiral of compact objects, starting with the breakthrough simulations of [1-3]. Rather accurate simulations of the last $\approx 10$ orbits of roughly equal mass binary black holes have become standard in the field (see e.g. [4-9] for recent examples). However, these simulations are still restricted to finite outer boundaries, and gravitational radiation is extracted at a number of finite shells. Maybe not surprisingly, it has been found that the wave extraction radius is a significant limiting factor for the precision of results [10-13]. The practical implementation of algorithms that include null infinity in the numerical grid is thus highly desirable. It also provides for a fruitful problem to foster the interaction of numerical and mathematical relativity. 
An important stepping stone to develop such algorithms is the case of linearized perturbations on a fixed background. The numerical study of linearized perturbations is convenient as one can evolve the gravitational radiation very accurately for a comparatively low computational cost [14-16]. For evolutions along null surfaces, this has provided important guidance in the past, see e.g. [17-19]. Note, however, that linear analysis may not be valid in certain special weak-field situations. Examples where the linear analysis breaks down have been found in studies of test fields [20,21]. In the pure gravitational case, so far it seems that the perturbative analysis fits well with the nonlinear evolution [15, 22-25].

When the background spacetime describes the particularly interesting case of a stationary black hole, the perturbation equations take the form of linear wave equations with a potential term (in our case the Bardeen-Press equation [26]). The solutions to such equations show three stages: an initial transient (the 'direct signal'), quasinormal mode ringing and power-law decay. The standard method to numerically analyze these stages in the time domain has been to solve initial boundary value problems using Cauchy-type foliations. This method has certain disadvantages. When one is primarily interested in radiative properties of the solution far away from the source, the use of Cauchy-type foliations is a waste of computational resources. Furthermore, there are difficulties related to the choice of boundary conditions. It has been shown that a typical choice of boundary data for numerical relativity destroys the polynomial tail behavior of solutions to wave equations on a Schwarzschild spacetime [27, 28]. Note that even if the polynomial tail can be calculated by using a better choice of boundary data, the decay rate will be the one near time-like infinity. It has been pointed out, however, that the relevant decay rate for an astrophysical observer is the one along null infinity [29-31].

Using foliations that approach null infinity would cure these problems. Numerical studies of gravitational perturbations at null infinity have been performed by $[17,18]$ using a characteristic approach for the Bardeen-Press equation, and have been compared with fully nonlinear studies in [19]. The authors have been able to efficiently calculate the quasinormal mode ringing and the power-law decay. There are, however, two main difficulties in the characteristic approach. An important aspect of solving the Bardeen-Press equation instead of the Regge-Wheeler-Zerilli equations is that the favorable separability properties of the Teukolsky formalism extends to Kerr spacetime [32]. Unfortunately, generalizing the studies of $[17,18]$ to Kerr spacetime is complicated because the construction of null foliations smoothly covering Kerr spacetime is not straightforward and from a numerical point of view not very convenient $[33,34]$. A further complication is related to the causal structure of a black-hole spacetime. Due to the global relationship between null surfaces on which boundary information is known to pose a characteristic initial value problem, the numerical solution for a perturbed black hole needs to be calculated in two steps: advanced [18] and retarded [17]. A related difficulty shows itself also in the choice of suitable coordinates which allow the computation of the gravitational waveform at null infinity and provide good resolution near the horizon.

An alternative and very flexible method to study radiation is to use spacelike surfaces that approach null infinity. Following [35], we call such surfaces hyperboloidal and the related initial value problem a hyperboloidal initial value problem. Hyperboloidal surfaces are as flexible as Cauchy-type surfaces, because the property that characterizes them is not local but global. Further, they enable, like characteristic surfaces, a clean treatment of gravitational radiation because they approach null infinity. In this sense, it is hoped that they combine the 'best of both worlds', and we expect increasing interest in this approach.

In the present paper, we want to learn from the linearized case as a preparation for the nonlinear hyperboloidal initial value problem. To this end, we construct solutions of the Bardeen-Press equation by numerically solving a hyperboloidal initial value problem for 
the perturbations of Schwarzschild spacetime and we calculate the gravitational wave signal at null infinity. We find that compactification along hyperboloidal surfaces leads to a very efficient code. Specifically, we show that the calculation of the quasinormal mode frequencies in the time domain can be performed accurately with a small number of grid points using standard numerical techniques. The last stage of the solution, namely the power-law decay, can be numerically calculated if the resolution is sufficiently high. A major advantage of our method for this part of the signal is not so much the efficiency but rather the fact that we can measure the decay rate at null infinity by solving a Cauchy problem in the PDE sense.

In the presentation and, to some extent, in the choice of the methods applied in the paper, we tried to avoid a heavy use of the conformal language. Our philosophy of constructing an algorithm for the hyperboloidal initial value problem follows the suggestions in [36, 37]. Instead of constructing a gauge-independent, regular, conformal Bardeen-Press equation, we make a suitable use of our gauge freedom before compactifying the coordinates and show that, in the end, regular equations are obtained. While both approaches are equivalent in their final aim, we hope that the way we present the method will be more accessible to numerical relativists who are not familiar with notions related to conformal geometry.

The paper is organized as follows. In section 2, we present the Teukolsky formalism and derive the Bardeen-Press equation for a spherically symmetric but otherwise arbitrary foliation. In section 3, we discuss our method to include null infinity on a numerical grid. We show that the hyperboloidal Bardeen-Press equation written in compactifying coordinates is regular at null infinity. After a short presentation of our numerical methods in section 4 , we go over to the numerical studies of the last two stages for the gravitational perturbations where we demonstrate some of the basic advantages of the hyperboloidal approach in numerical work. In section 5, we summarize our results and point out some ideas for future work.

\section{The perturbation equation}

\subsection{General definitions}

Teukolsky [32] derived a master perturbation equation for various fields in a Kerr background, using the spinor formalism introduced by Newman and Penrose [38]. One of the central ideas of this formulation consists in choosing a tetrad of null vectors, $Z_{a}{ }^{\mu}$, and then defining the directional operators as the projections of the covariant derivative along each null vector. For a complete review on the subject and its properties, the reader is referred to $[39,40]$.

Usually the tetrad is defined with four null vectors: two real and along the light cone, while the other two complex conjugate and in the perpendicular plane to the cone. There is much room for conventions regarding the orientation and scaling of the null vectors, and consequently for confusion. We make the following choices: we label the two real vectors along the light cone as $Z_{0}{ }^{\mu}=l^{\mu}, Z_{1}{ }^{\mu}=k^{\mu}$ and choose them both future directed with $l^{\mu}$ pointing outward and $k^{\mu}$ pointing inward. The complex two null vectors will be denoted by $m^{\mu}: Z_{2}^{\mu}=m^{\mu}, Z_{3}^{\mu}=m^{* \mu}$.

After these choices, there is still the issue of the normalization of the products $l^{\mu} k_{\mu}$ and $m^{\mu} m^{*}{ }_{\mu}$. The rest of the products are zero by construction. The properties mentioned above can be expressed in the following equations:

$$
Z_{a}{ }^{\mu} Z_{b \mu}=\eta_{a b}, \quad g_{\mu \nu}=2 \eta^{a b} Z_{a(\mu} Z_{|b| v)},
$$


with $\eta_{a b}$ being a matrix of the form

$$
\eta_{a b}=\left(\begin{array}{cccc}
0 & \eta_{12} & 0 & 0 \\
\eta_{12} & 0 & 0 & 0 \\
0 & 0 & 0 & -\eta_{12} \\
0 & 0 & -\eta_{12} & 0
\end{array}\right)
$$

with $\eta_{12}= \pm 1$ depending on the signature. If we choose the signature of the spacetime to be $(+,-,-,-)$ as in $[39,40]$, then the choice consistent with equations (1) to derive the perturbation equation is $\eta_{12}=1$ [32]. If the spacetime is described using the signature $(-,+,+,+)$, we have to choose $\eta_{12}=-1$ in order to remain consistent with equations (1). Some discussion on the change of the tetrad due to signature can be found in [41].

We derived the linearized equation for the perturbed Weyl scalar $\Psi_{4}$ (note that the background value vanishes), leaving unspecified the choice of signature. Using the definitions for the directional operators and spin coefficients as given in $[39,40]$, the final perturbation equation takes the form

$$
\begin{aligned}
\left(\left(\Delta+\eta_{12}\left(4 \mu_{s}\right.\right.\right. & \left.\left.+\mu_{s}^{*}+3 \gamma_{s}-\gamma_{s}^{*}\right)\right)\left(\mathbf{D}-\eta_{12}\left(\rho_{s}-4 \epsilon_{s}\right)\right)-\left(\delta^{*}+\eta_{12}\left(3 \alpha_{s}+\beta_{s}^{*}+4 \pi_{s}-\tau_{s}^{*}\right)\right) \\
& \left.\times\left(\delta+\eta_{12}\left(4 \beta_{s}-\tau_{s}\right)\right)-3 \eta_{12} \Psi_{2}\right) \Psi_{4}=0 .
\end{aligned}
$$

We have added a subindex ${ }_{s}$ to the spin coefficients in order to avoid confusion with other quantities that will be used later. In the rest of the work, we will be using the $(-,+,+,+)$ signature, and thus $\eta_{12}=-1$.

\subsection{Spherical symmetry}

We will work in spherically symmetric static spacetimes. Then the metric can be written as

$$
g=\left(-\alpha^{2}+\gamma^{2} \beta^{2}\right) \mathrm{d} t^{2}+2 \gamma^{2} \beta \mathrm{d} t \mathrm{~d} r+\gamma^{2} \mathrm{~d} r^{2}+r^{2} \mathrm{~d} \sigma^{2} .
$$

We will write the Bardeen-Press equation for $\Psi_{4}$ on such a background with respect to an orthonormal null tetrad $(l, k, m, \bar{m})$, consistent with the conditions mentioned above. The tetrad can be given by

$$
\begin{array}{rlrl}
l^{\mu} & =\frac{1}{2 \alpha^{2}}\left(1, \frac{\alpha}{\gamma}-\beta, 0,0\right), & & k^{\mu}=\left(1,-\left(\frac{\alpha}{\gamma}+\beta\right), 0,0\right), \\
m^{\mu} & =\frac{1}{\sqrt{2} r}(0,0,1, \mathrm{i} \csc \theta), & \bar{m}^{\mu}=\left(m^{\mu}\right)^{*} .
\end{array}
$$

Remember that $\Psi_{4}$ is defined by contracting the Weyl tensor with respect to the inward pointing null vector field of the null tetrad, $k$ in our case.

We compute the corresponding spin coefficients and directional derivatives and substitute into the perturbation equation (3). Using the definitions for the spin coefficients given in [39] and denoting derivatives with respect to $r$ by a prime, we obtain the nonzero spinors and Weyl scalars as

$$
\begin{aligned}
& \rho_{s}=\frac{\frac{\alpha}{\gamma}-\beta}{2 r \alpha^{2}} ; \quad \mu_{s}=\frac{\frac{\alpha}{\gamma}+\beta}{r} ; \quad \epsilon_{s}=-\frac{\left(r \alpha \gamma \rho_{s}\right)^{\prime}}{2 \alpha \gamma} ; \quad \gamma_{s}=-\frac{\left(r \alpha \gamma \mu_{s}\right)^{\prime}}{2 \alpha \gamma} ; \\
& \beta_{s}=-\frac{\cot \theta}{2 \sqrt{2} r} ; \quad \alpha_{s}=-\beta_{s}, \quad \Psi_{2}=\frac{\left(r^{2} \alpha \gamma \rho_{s} \mu_{s}\right)^{\prime}}{r \alpha \gamma} .
\end{aligned}
$$

Before proceeding to write down the perturbation equation for $\Psi_{4}$, we first note that the simple sourceless wave equation,

$$
g^{\mu \nu} \nabla_{\mu} \nabla_{\nu} \Phi\left(x^{\alpha}\right)=0,
$$


independently of the tensorial or spinorial character of $\Phi$ in this background takes the form

$$
\left[\mathcal{O}_{r t}+\mathcal{O}_{\theta \varphi}\right] \Phi=0
$$

with

$$
\begin{gathered}
\mathcal{O}_{r t}=\frac{r^{2}}{\alpha^{2}}\left(-\frac{\partial^{2}}{\partial t^{2}}+2 \beta \frac{\partial^{2}}{\partial t \partial r}+\left(\left(\frac{\alpha}{\gamma}\right)^{2}-\beta^{2}\right) \frac{\partial^{2}}{\partial r^{2}}+\frac{\alpha}{r^{2} \gamma}\left(\frac{r^{2} \beta \gamma}{\alpha}\right)^{\prime} \frac{\partial}{\partial t}\right. \\
\left.\quad+\frac{\alpha}{r^{2} \gamma}\left(r^{2}\left(\frac{\alpha}{\gamma}-\frac{\beta^{2} \gamma}{\alpha}\right)\right)^{\prime} \frac{\partial}{\partial r}\right), \\
\mathcal{O}_{\theta \varphi}=\frac{\partial^{2}}{\partial \theta^{2}}+\frac{1}{\sin ^{2} \theta} \frac{\partial^{2}}{\partial \varphi^{2}}+\cot \theta \frac{\partial}{\partial \theta} .
\end{gathered}
$$

In the perturbation equation (3), we use the sign consistent with our choice of signature, that is $\eta_{12}=-1$. After substituting our tetrad and the obtained directional operators, spin coefficients and the Weyl scalar $\Psi_{2}$, we obtain

$$
\left[L_{\mathrm{tr}}+L_{\theta \varphi}\right] \Psi_{4}=0
$$

where the operators $L_{\text {tr }}$ and $L_{\theta \varphi}$ are given by

$$
\begin{aligned}
& L_{\mathrm{tr}}=-\frac{r^{2}}{\alpha^{2}} \frac{\partial^{2}}{\partial t^{2}}+2 \beta \frac{r^{2}}{\alpha^{2}} \frac{\partial^{2}}{\partial t \partial r}+r^{2}\left(\frac{\alpha^{2}-\beta^{2} \gamma^{2}}{\alpha^{2} \gamma^{2}}\right) \frac{\partial^{2}}{\partial r^{2}}+A \frac{\partial}{\partial t}+B \frac{\partial}{\partial r}-2 r^{2} C, \\
& L_{\theta \varphi}=\mathcal{O}_{\theta \varphi}-4 \mathrm{i} \frac{\cos \theta}{\sin ^{2} \theta} \frac{\partial}{\partial \varphi}-2 \frac{\cos ^{2} \theta+1}{\sin ^{2} \theta} .
\end{aligned}
$$

Here, we have defined

$$
\begin{aligned}
A & =\frac{1}{\alpha \gamma}\left(\frac{r^{2} \beta \gamma}{\alpha}\right)^{\prime}-4 \frac{r^{4} \mu_{s}}{\alpha^{3}}\left(\frac{\alpha}{r}\right)^{\prime}, \\
B & =\frac{2}{\alpha \gamma}\left(r^{4} \alpha \gamma \rho_{s} \mu_{s}\right)^{\prime}-4 r^{2}\left(\frac{1}{\gamma^{2}}\left(\frac{\beta \gamma}{\alpha}\right)^{\prime}+2 r^{3} \frac{\rho_{s} \mu_{s}}{\alpha}\left(\frac{\alpha}{r}\right)^{\prime}\right), \\
C & =\frac{1}{r \alpha \gamma}\left(3\left(\alpha \gamma \rho_{s} \mu_{s} r^{2}\right)^{\prime}+\rho_{s} r\left(\alpha \gamma \mu_{s} r\right)^{\prime}-10 \mu_{s} r\left(\alpha \gamma \rho_{s} r\right)^{\prime}\right) \\
& \quad-\mu_{s}\left(5 \rho_{s}+r \rho_{s}{ }^{\prime}+2 r\left(\frac{\left(\alpha \gamma \rho_{s} r\right)^{\prime}}{\alpha \gamma}\right)^{\prime}\right)+\frac{2}{\alpha^{2} \gamma^{2}}\left(\alpha \gamma \rho_{s} r\right)^{\prime}\left(\alpha \gamma \mu_{s} r\right)^{\prime} .
\end{aligned}
$$

There are several things worth noticing in this equation. Its principal part is the same as of the wave equation. The gravitational contribution is described by some extra coefficients in the terms containing no higher than first radial derivatives. The left-hand side of the equation for the gravitational perturbation, as well as that for the wave equation, is separable into terms involving the $(r, t)$ and the angular coordinates. The equation is somewhat lengthy, due to the fact that we are considering general metric coefficients, but nevertheless, it is quite tractable. Finally, we remark that ignoring the difference between our signature convention and the one used in [32] would lead to an inconsistent expression.

We will work with the function $\widetilde{\Psi}_{4}=r \Psi_{4}$ in order to factor out the expected decay due to the peeling behavior. The function $\widetilde{\Psi}_{4}$ satisfies the equation

$$
\left[\widetilde{L}_{\mathrm{tr}}+L_{\theta \varphi}\right] \widetilde{\Psi}_{4}=0 \text {, }
$$


where the operator $\widetilde{L}_{\text {tr }}$ reads

$$
\widetilde{L}_{\mathrm{tr}}=-\frac{r^{2}}{\alpha^{2}} \frac{\partial^{2}}{\partial t^{2}}+2 \beta \frac{r^{2}}{\alpha^{2}} \frac{\partial^{2}}{\partial t \partial r}+2 r^{4} \rho_{s} \mu_{s} \frac{\partial^{2}}{\partial r^{2}}+\widetilde{A} \frac{\partial}{\partial t}+\widetilde{B} \frac{\partial}{\partial r}-2 r^{2} \widetilde{C},
$$

where now

$\widetilde{A}=A-2 r \frac{\beta}{\alpha^{2}}, \quad \widetilde{B}=B-4 r^{3} \rho_{s} \mu_{s}, \quad \widetilde{C}=C+\frac{B}{2 r^{3}}-2 \rho_{s} \mu_{s}$,

and $A, B$ and $C$ are given by (12). Further, using the fact that the Weyl scalar has spin weight $-2[39]$ to expand $\widetilde{\Psi}_{4}$ in terms of spin-weighted spherical harmonics

$$
\widetilde{\Psi}_{4}=\sum_{l m} \phi_{l, m}(t, r) Y_{-2}^{l, m}(\theta, \varphi) .
$$

To deal with spin-weighted harmonics, it is useful to introduce the so-called 'eth' and 'eth-bar' operators $[42,43]$ :

$$
\begin{aligned}
& \check{\partial}_{s}=-\left(\frac{\partial}{\partial \theta}+\mathrm{i} \csc \theta \frac{\partial}{\partial \varphi}-s \cot \theta\right) \equiv \varlimsup_{0}+s \cot \theta, \\
& \overline{\mathrm{d}}_{s}=-\left(\frac{\partial}{\partial \theta}-\mathrm{i} \csc \theta \frac{\partial}{\partial \varphi}+s \cot \theta\right) \equiv \bar{\partial}_{0}-s \cot \theta,
\end{aligned}
$$

which have the property of raising and lowering the spin weight of the function on which they act. It is straightforward to show that the angular operator in (11) can be expressed in terms of the eth operators as

$$
L_{\theta \varphi}=\bar{\partial}_{-1} \partial_{-2} .
$$

The $Y_{-2}^{l, m}$ are the eigenfunctions of this angular operator

$$
L_{\theta \varphi} Y_{-2}^{l, m}=\bar{\partial}_{-1} \partial_{-2} Y_{-2}^{l, m}=-(l-1)(l+2) Y_{-2}^{l, m} .
$$

In this way, the perturbation equation (13) transforms into a (1+1) equation for each $(l, m)$ mode of the gravitational perturbation,

$$
\left[\widetilde{L}_{\mathrm{tr}}-(l-1)(l+2)\right] \phi=0,
$$

where we have dropped the indices $l, m$. In our numerical work, we have used a first-order reduction of this equation as derived in the appendix.

\section{Including null infinity in the numerical domain}

In the previous section, we followed the standard procedure to calculate gravitational perturbations on a spherically symmetric background spacetime using the Teukolsky formalism with the additional feature that we allowed an arbitrary spherically symmetric foliation of the Schwarzschild background (see [44] for the corresponding generalization in the ReggeWheeler-Zerilli formalism). If we now insert the standard Schwarzschild gauge functions into equation (21) we get the familiar Bardeen-Press equation. Our aim, however, is to include null infinity in the numerical domain. In this section, we show how this can be done. 


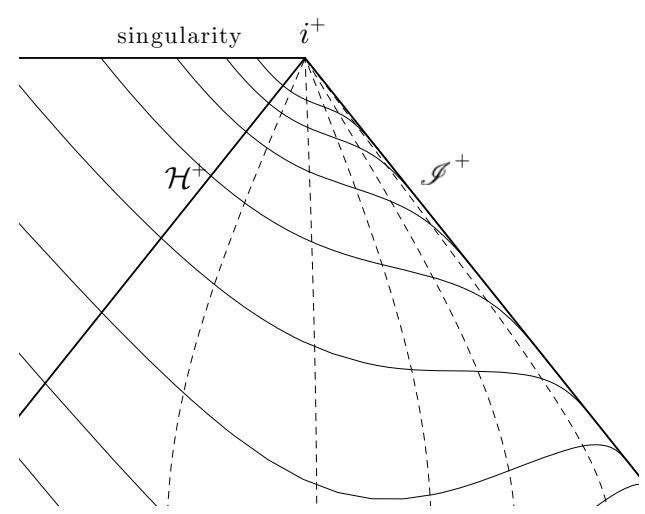

Figure 1. The Penrose diagram of a CMC foliation in Schwarzschild spacetime with $m=1 / 2$, $c=1, K=1$. The dashed lines represent Killing observers.

\subsection{Choice of a hyperboloidal foliation}

To include null infinity in the setting of a spacetime splitting, we first choose a hyperboloidal foliation by introducing a new time coordinate, $\tau$, whose level sets are hyperboloidal surfaces. Following [31], we consider the class of spherically symmetric constant mean curvature (CMC) foliations of Schwarzschild spacetime [45, 46]. The transformation from the standard Schwarzschild time coordinate to the time coordinate of a spherically symmetric CMC foliation can be written as $\tau=t-h(r)$, where $r$ is the Schwarzschild area radius, $h(r)$ is the height function and $t$ is the standard Schwarzschild time coordinate. The height function is not known in a closed form. Its radial derivative is given by

$$
\begin{aligned}
h^{\prime}(r) & =\frac{J(r)}{\left(1-\frac{2 m}{r}\right) P(r)}, \quad \text { where } \quad J(r)=\frac{K r}{3}-\frac{c}{r^{2}}, \quad \text { and } \\
P(r) & :=\sqrt{J(r)^{2}+\left(1-\frac{2 m}{r}\right)} .
\end{aligned}
$$

Here, the mass of the Schwarzschild black hole is denoted by $m$. The foliation parameters are the mean extrinsic curvature $K$, and a constant of integration $c$. The global behavior of CMC surfaces depends on the foliation parameters. We choose these parameters such that the surfaces of the foliation come from future null infinity, pass the event horizon above the bifurcation sphere and run into the future singularity as depicted in figure 1 [47]. The causal nature of the hyperboloidal foliation shown in the conformal diagram explains why we do not need a two-stage calculation of the waveform in contrast to the characteristic case $[17,18]$. A further convenient feature of this foliation is that the natural coordinates are adapted to time symmetry in the sense that the timelike Killing vector field of Schwarzschild spacetime $\partial_{t}$ is given by $\partial_{\tau}$ in the new coordinates. Therefore, timelike curves with constant spatial coordinates can be regarded as worldlines of natural observers at constant distances.

In coordinates adapted to the CMC slicing, the Schwarzschild metric is obtained in the form

$$
g=-\left(1-\frac{2 m}{r}\right) \mathrm{d} \tau^{2}-\frac{2 J(r)}{P(r)} \mathrm{d} \tau \mathrm{d} r+\frac{1}{P^{2}(r)} \mathrm{d} r^{2}+r^{2} \mathrm{~d} \sigma^{2}
$$


By comparing with (4) we see that the gauge functions read

$$
\alpha(r)=P(r), \quad \beta(r)=-J(r) \alpha(r), \quad \gamma(r)=\frac{1}{\alpha(r)} .
$$

An important difference to Cauchy foliations should be noted at this point. The asymptotic behavior of an asymptotically flat spacetime written in a Cauchy foliation implies $\alpha \sim 1$ and $\beta \sim 0$ as $r \rightarrow \infty$. The same spacetime written in a hyperboloidal foliation implies a different asymptotic behavior for the gauge functions, namely

$$
\alpha \sim O(r) \quad \text { and } \beta \sim O\left(r^{2}\right) \quad \text { as } \quad r \rightarrow \infty .
$$

In the following subsection, we will see that this behavior is important for the regularity of our equations.

\subsection{Compactification}

To include the asymptotic ends of the surfaces of our hyperboloidal foliation, we introduce a compactifying coordinate $\rho$ that is related to the standard Schwarzschild area-radius $r$ via

$$
r=\frac{\rho}{1-\rho}=\frac{\rho}{\Omega} \quad \text { where } \quad \Omega:=1-\rho .
$$

We choose the black-hole mass as $m=1 / 2$, the horizon is thus located at $\rho=1 / 2$. Future null infinity, $\mathscr{I}^{+}$, is located at $\rho=1$. Other choices are, of course, possible. Before writing the Bardeen-Press equation in the compactifying coordinate $\rho$, we factor out the singular asymptotic behavior of our hyperboloidal gauge functions (24) in order to deal with regular functions. Considering the asymptotic behavior of the conformal factor, $\Omega \sim 1 / r$, we define $\bar{\alpha}:=\Omega \alpha$ and $\bar{\beta}:=\Omega^{2} \beta$. The rescaled gauge source functions become

$\bar{\alpha}=\sqrt{\bar{J}^{2}+\left(1-\frac{2 m \Omega}{\rho}\right) \Omega^{2}}, \quad \bar{\beta}=-\bar{J} \bar{\alpha} \quad$ where $\quad \bar{J}:=\Omega J=\frac{K \rho}{3}-\frac{c \Omega^{3}}{\rho^{2}}$.

These functions are obviously regular at $\{\Omega=0\}$. Furthermore, they satisfy

$$
\left(\bar{\alpha}^{2}-\frac{\bar{\beta}^{2}}{\bar{\alpha}^{2}}\right)=O\left(\Omega^{2}\right) \quad \text { with }\left.\quad \beta\right|_{\mathscr{I}^{+}}<0 .
$$

We note that this behavior with the choice of the conformal factor (25) is specific to scri-fixing gauges and cannot be expected for all hyperboloidal foliations. It is related to the fact that the normal to level sets of $\tau$ lies in $\mathscr{I}^{+}$[47].

The definitions of the auxiliary variables given in (A.1) imply

$$
\psi=\partial_{r} \phi=\Omega^{2} \partial_{\rho} \phi=: \Omega^{2} \bar{\psi}, \quad \text { and } \quad \pi=\frac{1}{\alpha^{2}}\left(\partial_{t} \phi-\beta \partial_{r} \phi\right)=\frac{\Omega^{2}}{\bar{\alpha}^{2}}\left(\partial_{t} \phi-\bar{\beta} \bar{\psi}\right)=: \Omega^{2} \bar{\pi} .
$$

We write system (A.2) with respect to the functions $\bar{\alpha}, \bar{\beta}, \bar{\psi}, \bar{\pi}$ by factoring an $\Omega^{2}$ term out of the equations. The resulting first-order, symmetric hyperbolic system of equations reads

$$
\begin{aligned}
& \partial_{\tau} \phi=\bar{\alpha}^{2} \bar{\pi}+\bar{\beta} \bar{\psi} \\
& \partial_{\tau} \bar{\psi}=\partial_{\rho}\left(\bar{\alpha}^{2} \bar{\pi}+\bar{\beta} \bar{\psi}\right), \\
& \partial_{\tau} \bar{\pi}=\partial_{\rho}\left(\bar{\alpha}^{2} \bar{\psi}+\bar{\beta} \bar{\pi}\right)+A_{\pi} \bar{\pi}+A_{\psi} \bar{\psi}+\left(\frac{A_{\phi}}{\Omega^{2}}-\frac{\lambda}{\rho^{2}}\right) \phi,
\end{aligned}
$$




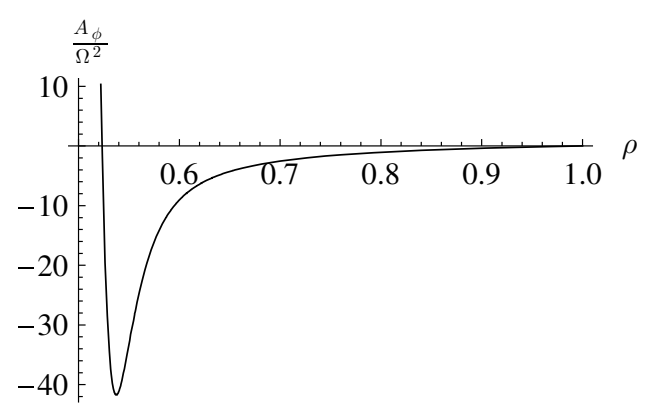

Figure 2. The function $A_{\phi} / \Omega^{2}$ from (28) for the same hyperboloidal foliation as in the diagram. We see that $C / \Omega^{2}$ vanishes at null infinity.

where $A_{\pi}, A_{\psi}$ and $A_{\phi}$ are functions of $\rho$. It turns out that $A_{\pi}$ and $A_{\psi}$ are conformally covariant. Writing $A_{\pi}$ and $A_{\psi}$ from (A.3) in terms of the rescaled gauge functions and the compactifying coordinate $\rho$ results in

$$
A_{\pi}=-4\left(\bar{\alpha}^{2}+\bar{\beta}\right) \partial_{\rho}\left(\ln \frac{\bar{\alpha}}{\rho}\right), \quad A_{\psi}=A_{\pi}-4 \bar{\alpha}^{2} \partial_{\rho}\left(\frac{\bar{\beta}}{\bar{\alpha}^{2}}\right) .
$$

To show the regularity of our equations, we need to show that $A_{\phi}$ falls off with $\Omega^{2}$. We have by (A.3)

$$
\begin{gathered}
\frac{A_{\phi}}{\Omega^{2}}=-\frac{\Omega}{\rho} \partial_{\rho}\left(\frac{1}{\Omega^{2}}\left(\bar{\alpha}^{2}-\frac{\bar{\beta}^{2}}{\bar{\alpha}^{2}}\right)\right)+\frac{4}{\Omega \rho}\left(\bar{\alpha}^{2} \partial_{\rho}\left(\frac{\bar{\beta}}{\bar{\alpha}^{2}}\right)+\left(\bar{\alpha}^{2}-\frac{\bar{\beta}^{2}}{\bar{\alpha}^{2}}\right) \partial_{\rho}\left(\ln \frac{\bar{\alpha}}{\rho}\right)\right) \\
+2 \frac{\left(\bar{\alpha}^{2}+\bar{\beta}\right)^{2}}{\Omega^{2} \rho^{2}} \partial_{\rho}\left(\frac{\Omega^{4} \partial_{\rho}\left(\frac{\rho^{2}}{\Omega^{2}}\left(1-\frac{\bar{\beta}}{\bar{\alpha}^{2}}\right)\right)}{\bar{\alpha}^{2}+\bar{\beta}}\right) .
\end{gathered}
$$

The explicit regularity of the above expression follows from the behavior of our rescaled gauge functions (27), specifically from $\bar{\alpha}^{2}+\bar{\beta} \sim O\left(\Omega^{2}\right)$ near null infinity. Indeed, as we can see in figure 2 the expression on the right-hand side of (28) is not just regular but vanishes at null infinity for the foliation given in (26). We note that scri-fixing plays an essential role here.

\section{Numerical studies}

\subsection{Initial data}

As we are not interested in the first (transient) part of the signal, the initial data can be chosen quite freely. We take a simple Gaussian as the initial perturbation and set

$$
\phi(0, \rho)=a \mathrm{e}^{-\left(\rho-\rho_{c}\right)^{2} / \sigma^{2}}, \quad \psi(0, \rho)=\partial_{\rho} \phi(0, \rho), \quad \pi(0, \rho)=0 .
$$

Here, $\rho_{c}$ is the center of the Gaussian pulse, $a$ is its amplitude and $\sigma$ is its width. We set $\rho_{c}=0.7, a=1$ and $\sigma=0.05$ on the domain $\rho \in[0.495,1]$. The solid line in figure 7 shows the initial data. We have tested that different choices of initial data do not have a relevant influence on the features we discuss in the following. Note that the vanishing of $\pi(0, r)$ does not mean that the data are time symmetric, because the initial surface is future hyperboloidal (spacelike while approaching future null infinity). We focus on the typically dominant $l=2$ mode, corresponding to the lowest allowed angular momentum number for a spin-2 field because higher modes are weaker due to their faster decay rates and longer quasinormal mode ringing phases. 


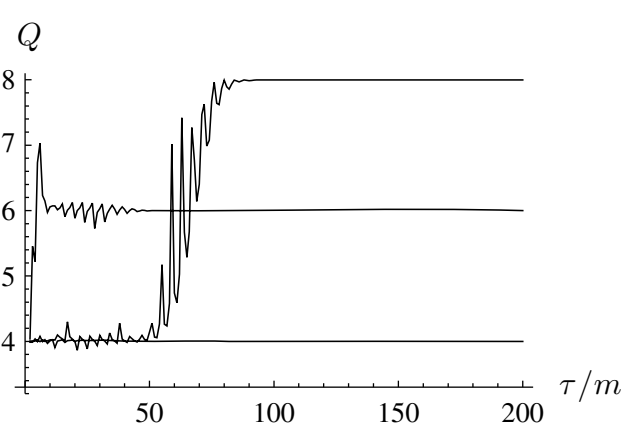

Figure 3. Convergence in the $L_{2}$-norm indicating fourth-, sixth- and eighth-order convergence for the corresponding finite difference operators. The convergence factor $Q$ is calculated by $Q=\log _{2} \frac{\left\|\phi^{\text {low }}-\phi^{\text {med }}\right\|}{\left\|\phi^{\text {med }}-\phi^{\text {high }}\right\|}$.

\subsection{Numerical algorithms}

We adopt the method of lines approach and discretize time and space separately, using fourthorder Runge-Kutta time integration and finite differencing with fourth-, sixth- and eighth-order accurate stencils. We add Kreiss-Oliger-type artificial dissipation to the evolution equation for $\pi$ to suppress numerical high-frequency waves [48]. For a $2 p-2$ accurate scheme, we choose an operator $(Q)$ of order $2 p$ as

$$
Q=\epsilon(-1)^{p} \frac{h^{2 p-1}}{2^{p}} D_{+}^{p} D_{-}^{p},
$$

where $h$ is the grid size, $D_{ \pm}$are the forward and backward finite differencing operators and $\epsilon$ is the dissipation parameter. Unless otherwise stated, we set $\epsilon=0.07$.

The inner boundary is a spacelike surface inside the event horizon where no boundary data are required. We excise the interior domain near the singularity. For the class of CMC surfaces we have chosen, excision is necessary as there is a minimal surface inside the event horizon where our coordinates break down. The outer boundary is at null infinity where one-sided finite differencing is applied. The one-sided finite differencing is of the same order as the interior one.

The convergence of the code can be seen in figure 3 . For this plot, a three-level convergence analysis has been performed with 505-, 1010- and 2020-grid cells using finite difference operators of fourth, sixth and eighth order. For the sixth- and eighth-order convergence tests, we used quadruple precision, because the numerical error was below machine accuracy at late times when double precision was used. At very late times, convergence is lost due to the accumulation of numerical errors (see the bending down of the curves in figure 9 at late times).

Figure 4 shows the $L_{2}$-norm of the relative errors between the above-mentioned simulations in different resolutions and finite differencing orders. Using an eighth-order accurate finite differencing improves the final error by eight orders of magnitude in the high-resolution runs confirming that high accuracies can be achieved with high-order finite differencing in combination with a relatively small amount of grid points.

The simulation domain in the compactifying coordinate reads $\rho \in[0.495,1]$. The Courant factor $\Delta t / \Delta \rho$ in the simulations has been chosen to be 4 . We can choose a Courant factor that is larger than 1 , because the coordinate speeds of characteristics are less than 1. For outgoing and ingoing characteristics, the characteristic speeds with respect to our foliation are $v_{ \pm}= \pm \bar{\alpha}^{2}-\bar{\beta}$. Figure 5 shows the characteristic speeds along the grid for $K=1$. 


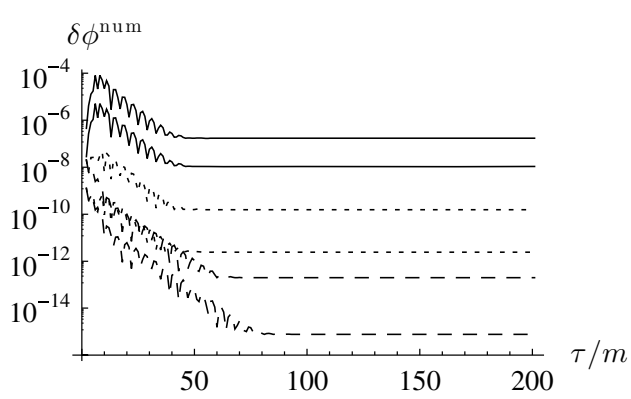

Figure 4. $L_{2}$-norm of the differences between numerical solutions obtained using different resolutions, i.e. $\left\|\phi^{\text {low }}-\phi^{\text {med }}\right\|$ and $\left\|\phi^{\text {med }}-\phi^{\text {high }}\right\|$. Solid curves denote errors for fourth-order finite difference operators, pointed curves for sixth-order and dashed curves for eighth order.

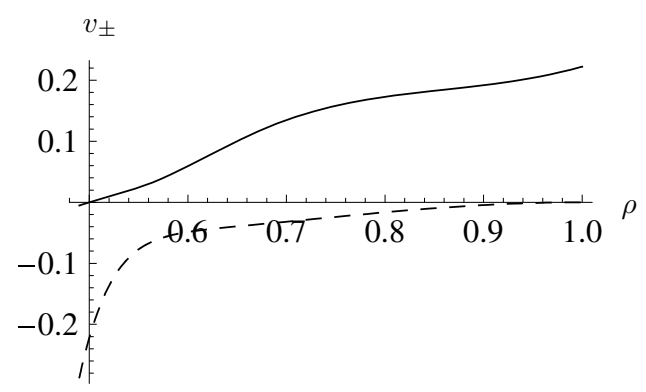

Figure 5. The dashed and the solid curves denote coordinate speeds of in- and outgoing characteristics respectively, for the foliation characterized by (26). The horizon is located at $\rho=0.5$. We see that the characteristic speeds are less than unity which allows us to choose a large Courant factor. We observe that there is little variation of the speeds over the grid which has infinite physical extent.

The allowed Courant factor depends on the value of $K$, as $K$ is related to the coordinate speed of characteristics. For example, the coordinate speed of outgoing characteristics at $\mathscr{I}^{+}$reads in our foliation $v_{+} \mid \mathscr{I}^{+}=2 K^{2} / 9$. Therefore, a large value for $K$ implies a small Courant factor [47]. We plot in figure 5 the speeds for $K=1$ and see that $v_{ \pm}<1$, which allows $\Delta t / \triangle \rho>1$. We also see that the ingoing characteristic speed vanishes at $\mathscr{I}^{+}$as expected, correspondingly we do not need to impose outer boundary conditions. Note also that there is relatively little variation of the characteristic speeds over this grid which has infinite physical extent.

In the studies presented below, we choose $m=1 / 2, c=1$ and $K=1$.

\subsection{Quasinormal mode ringing}

Figure 6 shows the gravitational perturbations of a Schwarzschild black hole in a double logarithmic plot with respect to three observers located at $r \approx\{2.5 \mathrm{~m}, 18 \mathrm{~m}\}$ and at $\mathscr{I}^{+}$. We see that, as expected, the quasinormal mode frequencies are the same for each observer. It does not matter for the calculation of these frequencies at what distance from the black hole the perturbations are measured. The end of the quasinormal ringing phase, however, is observer dependent. The polynomially decaying part of the solution starts earlier for an observer at $\mathscr{I}^{+}$than for a nearby observer. This suggests that for the calculation of quasinormal mode 


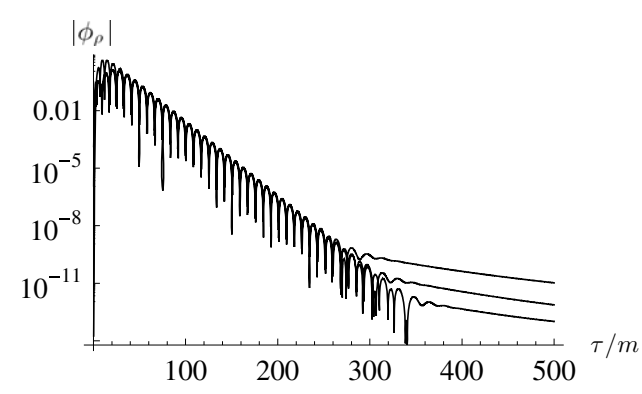

Figure 6. The quasinormal mode ringing of Schwarzschild spacetime excited by a Gaussian gravitational perturbation with $l=2$ measured by three observers. Looking to the tail part of the signal, the observers are located from top to bottom at $\mathscr{I}^{+}, r \approx 18 \mathrm{~m}$ and $r \approx 2.5 \mathrm{~m}$. We see that the ringing phase ends at different times for different observers and goes into a polynomial decay.

Table 1. Numerical quasinormal mode frequencies $\omega_{i}^{\text {num(2p) }}$ in various resolutions measured at $r \approx 2.5 \mathrm{~m}$ and the relative errors $\delta \omega_{i}^{(2 p)}$ calculated in a numerical simulation with spatial finite difference operators of the order $2 p$. We perform a simple least square fit to (29) with initial guess. We see that even with low resolutions, we are able to measure the quasinormal-mode frequencies with a high accuracy indicating the efficiency of our method.

\begin{tabular}{rllllllll}
\hline Cells & $\omega_{1}^{\text {num(4) }}$ & $\omega_{2}^{\text {num(4) }}$ & $\delta \omega_{1}^{(4)}$ & $\delta \omega_{2}^{(4)}$ & $\omega_{1}^{\text {num(6) }}$ & $\omega_{2}^{\text {num(6) }}$ & $\delta \omega_{1}^{(6)}$ & $\delta \omega_{2}^{(6)}$ \\
\hline $25^{\mathrm{a}}$ & 0.825455 & 0.157916 & $10^{-2}$ & $1.1 \times 10^{-2}$ & 0.777778 & 0.168439 & $4.1 \times 10^{-2}$ & $5.3 \times 10^{-2}$ \\
50 & 0.746988 & 0.177897 & $4.7 \times 10^{-4}$ & $1.5 \times 10^{-4}$ & 0.747341 & 0.177917 & $2.3 \times 10^{-6}$ & $4.4 \times 10^{-5}$ \\
100 & 0.747325 & 0.177927 & $2.4 \times 10^{-5}$ & $1.1 \times 10^{-5}$ & 0.747344 & 0.177923 & $1.9 \times 10^{-6}$ & $9.5 \times 10^{-6}$ \\
200 & 0.747343 & 0.177924 & $2.6 \times 10^{-7}$ & $4.9 \times 10^{-6}$ & 0.747344 & 0.177924 & $1.8 \times 10^{-6}$ & $5.4 \times 10^{-6}$ \\
\hline
\end{tabular}

${ }^{\text {a }}$ For the runs with 25 grid cells, a relatively high dissipation parameter of $\epsilon=0.2$ has been used.

frequencies, one may get better results by using the waveform as measured by a nearby observer.

We expect the quasinormal mode ringing to have the form

$$
\phi_{\rho}(\tau)=a_{\rho} \mathrm{e}^{-\omega_{2} \tau} \sin \left(\omega_{1} \tau+\varphi_{\rho}\right) .
$$

Here, $\omega_{1}$ and $\omega_{2}$ are the mode frequencies, $a_{\rho}$ is the amplitude and $\phi_{\rho}$ is the phase of the wave signal. In contrast to the mode frequencies, the amplitude and the phase do depend in general on the observer's location.

To demonstrate the efficiency of our method, we calculate the quasinormal mode frequencies during the ringing phase using various resolutions. The frequencies are compared to the result from Leaver's continued fraction method [49]. For our choice of black-hole mass of $m=1 / 2$, they read $\left(\omega_{1}, \omega_{2}\right)=(0.747343,0.177925)$. Table 1 shows the measured frequencies for various grid resolutions for fourth- and sixth-order finite differencing operators along with the relative errors. The relative error $\delta \omega_{i}^{(2 p)}$ for $\omega_{i}^{\text {num(2p) }}$ in a numerical simulation with spatial finite differencing of order $2 p$ is calculated by $\delta \omega_{i}^{(2 p)}=\left|\omega_{i}^{\text {num }(2 p)}-\omega_{i}\right| / \omega_{i}$. The fitting is performed using a simple least-squares method on the interval $\tau \in[80 \mathrm{~m}, 180 \mathrm{~m}]$ with an initial guess of the frequencies.

We see that already with 50 cells we are able to calculate the quasinormal mode frequencies with a relative error of about $10^{-4}$ using fourth-order finite differences and $10^{-6}$ using sixthorder finite differences. The use of higher order accurate finite differencing does not increase the computational cost significantly. We observed only about a $5 \%$ increase in the run time for 


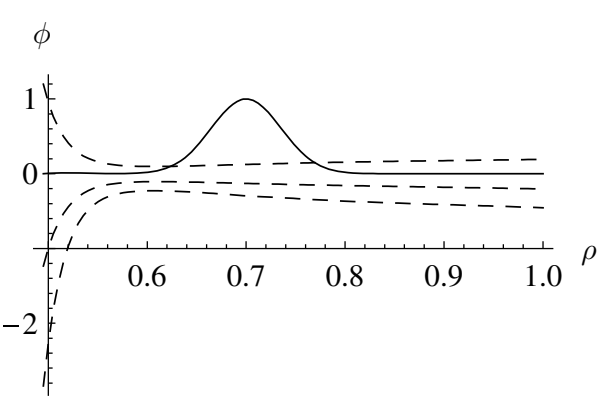

Figure 7. The numerical solution on the grid during the quasinormal ringing phase plotted at different times and rescaled for visibility. The solid curve is just the initial data. The dashed curves are from top to bottom: $\{10 \times \phi(80 m, \rho), 100 \times \phi(106 m, \rho), 1000 \times \phi(122 m, \rho)\}$. We see that the wave package is distributed quite uniformly across the grid.

the sixth-order finite differencing compared to the fourth-order one. We note that for a relative error at the order of $10^{-6}$, the accuracy in the frequency is rather dominated by the fit than by numerics. This explains, in part, why the relative error in the simulation with 200-grid cells seems smaller in the fourth-order case than in the sixth-order case. Using eighth-order finite differencing gives similar results as the sixth-order one and does not increase the accuracy in the frequencies considerably.

The fact that using a very small number of cells is already sufficient for such accuracy in the frequencies demonstrates the efficiency of the hyperboloidal approach. An explanation for this efficiency can be found in the way the solution looks like. Figure 7 shows the solution at three different times during the quasinormal ringing phase. We see that the initially localized perturbation is distributed uniformly on the grid. This uniform distribution implies that there is no need to use a high number of grid points to resolve the outgoing wave package. For accuracy, it is more efficient to use a high-order scheme with a small number of grid points in this case. In evolutions with Cauchy-type foliations, however, the outgoing wave package is a long wavetrain with the typical quasinormal ringing form that requires good resolution. We note that the observed broadening in the hyperboloidal approach is stronger for larger values of $K$. For smaller values, we get a similar localization like when Cauchy-type foliations are used. This is because $K$ can be regarded as a measure of how close the behavior of hyperboloidal surfaces is to being characteristic [47].

\subsection{Polynomial decay}

Figure 8 shows the polynomially decaying part of the solution starting from $\tau=500 \mathrm{~m}$ as seen at future null infinity, $\mathscr{I}^{+}$, at $r \approx 60 \mathrm{~m}$ and at the future horizon, $\mathcal{H}^{+}$, in an evolution with 4040-grid cells. We observe that the scattered perturbations are stronger for the observer at $\mathscr{I}^{+}$and also the slope of the curves are different, indicating that the decay rate for a far away observer is less than the decay rate for a close by observer. An intuitive explanation for this difference is given by the picture that the tail is generated by backscattering off the nonvanishing curvature of the background spacetime. An observer who is farther away sees more scattering than a nearby observer. Therefore, the amount of scattered waves measured by a nearby observer is less than for a farther one; consequently, the scattered part of the signal is 'stronger' for the far away observer (modulo the natural fall-off) and decays slower. The 


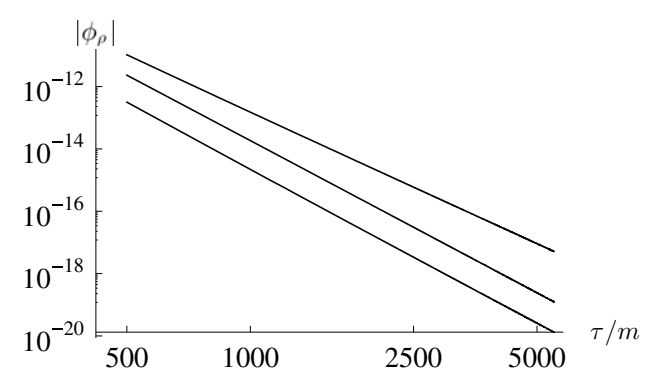

Figure 8. The polynomially decaying part of the solution in a double-logarithmic plot starting from $\tau=500 \mathrm{~m}$. The upper curve gives the solution at null infinity, the middle one at $r \approx 60 \mathrm{~m}$ and the lower one at the future horizon $\mathcal{H}^{+}$. It can be seen that the decay of the solution along $\mathscr{I}^{+}$ is slower than near the black hole.

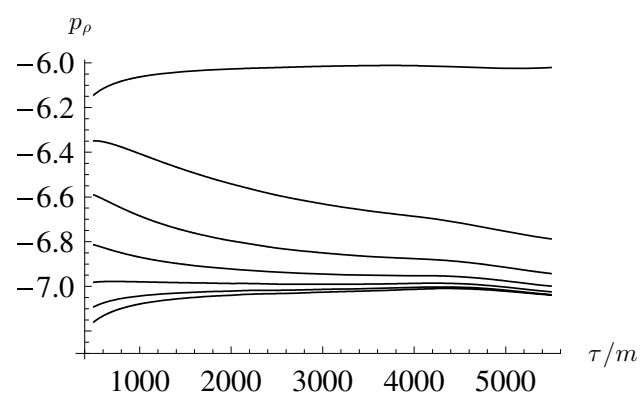

Figure 9. The local power index $p_{\rho}(\tau)$ for observers located from top to bottom at $\left\{\mathscr{I}^{+}, 800 m, 260 m, 110 m, 50 m, 25 m\right\}$. We see that after about $\tau=4500 \mathrm{~m}$ the local power index for nearby observers bends down. This behavior is seen earlier for lower resolutions and is only a numerical effect indicating loss of convergence due to loss of accuracy.

natural fall-off is not seen in figure 8 , as the variable we plot corresponds to the real part of $\Psi_{4}$ rescaled with $r$.

We see from figures 6 and 8 that we are able to follow the solution for more than 20 orders of magnitude and calculate the predicted decay rate with high accuracy. To achieve such accuracy, we use eighth-order finite difference operators. The exponentially decaying (quasinormal mode ringing) part of the solution is calculated in quadruple precision. The reason for choosing eighth-order accurate finite differencing is due to the very small effects that we are trying to measure. Note that for any given finite difference order, there are ghost potentials which may be mistaken as tail solutions [50]. We have tested that the tail seen in figure 8 is not a numerical artifact by comparing solutions in various resolutions.

An accurate picture of the decay rates can be obtained from figure 9. Here we plot, for various observers, the local power index defined as

$$
p_{\rho}(\tau)=\frac{\mathrm{d} \ln |\phi(\tau, \rho)|}{\mathrm{d} \ln \tau}
$$

The function $p_{\rho}(\tau)$ becomes asymptotically the exponent of the polynomial decay of the solution. At late times, our numerically measured decay rate becomes -6.01 for an observer at $\mathscr{I}^{+}$and -7.002 for an observer near $i^{+}$, in accordance with the well-known prediction of $2^{-(2 l+2)}$ at $\mathscr{I}^{+}$and of $2^{-(2 l+3)}$ near $i^{+}[51]$ (see also [29, 52-57]). 


\section{Discussion}

In this paper, we have revisited the problem of scattering pulses of gravitational waves off a Schwarzschild black hole with the aim of getting guidance in the development of a hyperboloidal approach to numerical relativity. We have used constant mean curvature slices on the background of a Schwarzschild spacetime [45, 46] in a scri-fixing gauge [47] to numerically solve a hyperboloidal initial value problem [31] for the Bardeen-Press equation. The numerical study of gravitational perturbations at null infinity follows, in some sense, the spirit of [17-19] who studied compactified null foliations.

Motivation for taking a hyperboloidal approach comes from the well-known shortcomings of existing methods based on Cauchy-type and characteristic foliations for this problem. The drawbacks of using Cauchy-type foliations can be summarized as follows: (i) waste of computational resources when one is interested in the waveform as seen by far away observers, (ii) spurious reflections from the outer boundary contaminating the waveform in long-time evolutions due to unknown boundary data, (iii) not being able to read off the waveform at null infinity. Using a characteristic foliation solves these problems, but leads to new difficulties: (i) the causal relationship between the surfaces seems to require a two-stage approach $[18,17]$, (ii) choice of variables for the numerical simulations is not straightforward due to loss of resolution, and most importantly, (iii) extension beyond spherical symmetry is very complicated. Especially in highly dynamical situations where formation of caustics is expected [58], it is not yet clear how the characteristic approach can be implemented [59].

The hyperboloidal approach hopes to combine the best properties of currently used approaches in the field, namely the flexibility of Cauchy-type surfaces and the asymptotic behavior of characteristic surfaces, and avoids their problems - however it is also much less developed so far.

We believe that the problem of treating gravitational perturbations on a fixed spherically symmetric background, while rather simple, is an important step in the development of algorithms for the hyperboloidal initial value problem. We have chosen to solve the Bardeen-Press equation describing perturbations of Schwarzschild spacetime in terms of the Weyl component $\psi_{4}$, partly because this naturally generalizes to the much more interesting axisymmetric case via the Teukolsky equation.

We demonstrated in this paper that compactification along hyperboloidal surfaces using the Teukolsky formalism on the background of a Schwarzschild spacetime leads to regular equations. This regular behavior can be expected on the background of any weakly asymptotically simple spacetime (in the sense of Penrose [60, 61]). The resulting equations can be solved numerically to study quasinormal mode ringing very efficiently. The tail part can be calculated accurately with sufficient numerical resolution. There are no reflections from the numerical outer boundary. The only approximation we use to calculate the gravitational waveform beside the linearization is the numerical discretization of the equations. The observed uniform distribution of outgoing wave packages along the grid and the numerical experiments we performed suggest that the use of high-order methods to solve the linear perturbation equations lead to an efficient code that requires a moderate number of grid points while achieving good accuracy in the results.

We want to conclude with a brief discussion of open problems for future work. It may be interesting to compare the approach for curvature-based and metric perturbations using the Chandrasekhar transformation along the lines of [14]. Current work is under way to study metric perturbations using the Regge-Wheeler-Zerilli formalism in a hyperboloidal setting [62]. The hyperboloidal approach to study gravitational perturbations at null infinity should 
generalize rather straightforwardly to Kerr spacetime, for example, by using the foliations presented in [47].

The difficulty of generalization to the fully nonlinear Einstein equations remains unclear. In contrast to the test field case, the conformally rescaled Einstein equations are not explicitly regular. There, a scri-fixing gauge can be constructed in a hyperboloidal initial value problem for the generalized harmonic reduction of the Einstein equations such that the formally singular terms attain regular limits at null infinity [63]. The open question is whether such limits can be calculated numerically in a stable way. The asymptotic behavior of the gauge functions (27) need to be taken into account if a regularization along the lines of [63] needs to be done for the ADM or BSSN systems based on scri-fixing. A very promising alternative is to adopt an elliptic-hyperbolic formulation of the problem, and apply boundary conditions for gauge quantities as an elliptic Dirichlet problem, as suggested in [37]. If the nonlinear problem can be treated successfully, even for the spherically symmetric case, it would be interesting to study questions concerning, fow example, nonlinear propagation of waves to null infinity or the conservation of Newman-Penrose quantities which has been the focus in the original work of Bardeen and Press [26].

\section{Acknowledgments}

This work was supported in part by the NSF grant PHY0801213 to the University of Maryland and by the DFG grant SFB/Transregio 7 'Gravitational Wave Astronomy'. S Husa is a VESF fellow of the European Gravitational Observatory (EGO). D Núñez is grateful to Luciano Rezzolla for warm hospitality during his stay at the AEI, and acknowledges DAAD, DGAPAUNAM and CONACyT grants for partial support.

\section{Appendix A. Symmetric hyperbolic form of the Bardeen-Press equation}

We solve the Bardeen-Press equation in a first-order symmetric hyperbolic form using a spherically symmetric CMC foliation of Schwarzschild spacetime with the gauge functions given in (23). The relation $\alpha=1 / \gamma$ simplifies the calculation, so we will assume it in the following. In such a gauge, the spin coefficients take the form

$$
\rho_{s}=\frac{\alpha^{2}-\beta}{2 \alpha^{2} r}, \quad \mu_{s}=\frac{\alpha^{2}+\beta}{r} .
$$

To bring the radial part of the Bardeen-Press equation (21) to the first-order form, we introduce the auxiliary variables

$$
\psi:=\partial_{r} \phi, \quad \text { and } \quad \pi:=\frac{1}{\alpha^{2}}\left(\partial_{t} \phi-\beta \partial_{r} \phi\right) .
$$

Then the following linear, symmetric hyperbolic system of evolution equations is obtained from (14), (15)

$$
\begin{aligned}
& \partial_{t} \phi=\alpha^{2} \pi+\beta \psi \\
& \partial_{t} \psi=\partial_{r}\left(\alpha^{2} \pi+\beta \psi\right), \\
& \partial_{t} \pi=\partial_{r}\left(\alpha^{2} \psi+\beta \pi\right)+A_{\pi} \pi+A_{\psi} \psi+\left(A_{\phi}-\frac{\lambda}{r^{2}}\right) \phi,
\end{aligned}
$$




$$
\begin{aligned}
& \text { with } \lambda=(l-1)(l+2) \text { and } \\
& \begin{array}{c}
A_{\pi}=-4\left(\alpha^{2}+\beta\right) \partial_{r}\left(\ln \frac{\alpha}{r}\right) \\
A_{\psi}=A_{\pi}-4 \alpha^{2} \partial_{r}\left(\frac{\beta}{\alpha^{2}}\right) \\
A_{\phi}=-\frac{1}{r} \partial_{r}\left(\alpha^{2}-\frac{\beta^{2}}{\alpha^{2}}\right)+\frac{4}{r}\left(\alpha^{2} \partial_{r}\left(\frac{\beta}{\alpha^{2}}\right)+\left(\alpha^{2}-\frac{\beta^{2}}{\alpha^{2}}\right) \partial_{r}\left(\ln \frac{\alpha}{r}\right)\right) \\
\quad+\frac{2\left(\alpha^{2}+\beta\right)^{2}}{r^{2}} \partial_{r}\left(\frac{\partial_{r}\left(r^{2}\left(1-\frac{\beta}{\alpha^{2}}\right)\right)}{\alpha^{2}+\beta}\right)
\end{array}
\end{aligned}
$$

\section{References}

[1] Pretorius F 2005 Evolution of binary black hole spacetimes Phys. Rev. Lett. 95121101

[2] Campanelli M, Lousto C O, Marronetti P and Zlochower Y 2006 Accurate evolutions of orbiting black-hole binaries without excision Phys. Rev. Lett. 96111101

[3] Baker J G, Centrella J, Choi D-Il, Koppitz M and van Meter J 2006 Gravitational wave extraction from an inspiraling configuration of merging black holes Phys. Rev. Lett. 96111102

[4] Hannam M, Husa S, Brügmann B and Gopakumar A 2008 Comparison between numerical-relativity and post-Newtonian waveforms from spinning binaries: the orbital hang-up case Phys. Rev. D 78104007

[5] Hinder I, Herrmann F, Laguna P and Shoemaker D 2008 Comparisons of eccentric binary black hole simulations with post-Newtonian models arXiv:0806.1037

[6] Campanelli M, Lousto C O, Nakano H and Zlochower Y 2008 Comparison of numerical and post-Newtonian waveforms for generic precessing black-hole binaries arXiv:0808.0713

[7] Boyle M et al 2008 High-accuracy numerical simulation of black-hole binaries: computation of the gravitationalwave energy flux and comparisons with post-Newtonian approximants arXiv:0804.4184

[8] Baker J G et al 2008 Mergers of non-spinning black-hole binaries: Gravitational radiation characteristics Phys. Rev. D 78044046

[9] Pollney D et al 2007 Recoil velocities from equal-mass binary black-hole mergers: a systematic investigation of spin-orbit aligned configurations Phys. Rev. D 76124002

[10] Hannam M, Husa S, Sperhake U, Brügmann B and González J A 2008 Where post-Newtonian and numericalrelativity waveforms meet Phys. Rev. D 77044020

[11] Boyle M et al 2007 High-accuracy comparison of numerical relativity simulations with post-Newtonian expansions Phys. Rev. D 76124038

[12] Pazos E, Dorband E N, Nagar A, Palenzuela C, Schnetter E and Tiglio M 2007 How far away is far enough for extracting numerical waveforms, and how much do they depend on the extraction method? Class. Quantum Grav. 24 S341

[13] Kocsis B and Loeb A 2007 Distortion of gravitational-wave packets due to their self-gravity Phys. Rev. D 76084022

[14] Campanelli M, Krivan W and Lousto C O 1998 The imposition of Cauchy data to the Teukolsky equation: II. Numerical comparison with the Zerilli-Moncrief approach to black hole perturbations Phys. Rev. D 58024016

[15] Baker J G, Campanelli M and Lousto C O 2002 The Lazarus project: a pragmatic approach to binary black hole evolutions Phys. Rev. D 65044001

[16] Pazos-Avalos E and Lousto C O 2005 Numerical integration of the Teukolsky equation in the time domain Phys. Rev. D 72084022

[17] Husa S, Zlochower Y, Gomez R and Winicour J 2002 Retarded radiation from colliding black holes in the close limit Phys. Rev. D 65084034

[18] Campanelli M, Gomez R, Husa S, Winicour J and Zlochower Y 2001 The close limit from a null point of view: the advanced solution Phys. Rev. D 63124013

[19] Zlochower Y, Gomez R, Husa S, Lehner L and Winicour J 2003 Mode coupling in the nonlinear response of black holes Phys. Rev. D 68084014

[20] Bizon P, Chmaj T and Rostworowski A 2007 On asymptotic stability of the Skyrmion Phys. Rev. D 75121702

[21] Bizon P, Chmaj T and Rostworowski A 2007 Late-time tails of a Yang-Mills field on Minkowski and Schwarzschild backgrounds Class. Quantum Grav. 24 F55 
[22] Baker J G et al 2000 Nonlinear and perturbative evolution of distorted black holes. II. Odd-parity modes Phys. Rev. D 62127701

[23] Baker J G, Campanelli M, Lousto C O and Takahashi R 2002 Modeling gravitational radiation from coalescing binary black holes Phys. Rev. D 65124012

[24] Baker J G, Campanelli M, Lousto C O and Takahashi R 2004 Coalescence remnant of spinning binary black holes Phys. Rev. D 69027505

[25] Campanelli M, Kelly B J and Lousto C O 2006 The Lazarus project: II. Space-like extraction with the quasiKinnersley tetrad Phys. Rev. D 73064005

[26] Bardeen J M and Press W H 1973 Radiation fields in the Schwarzschild background J. Math. Phys. 14 7-19

[27] Allen E W, Buckmiller E, Burko L M and Price R H 2004 Radiation tails and boundary conditions for black hole evolutions Phys. Rev. D 70044038

[28] Dafermos M and Rodnianski I 2004 A note on boundary value problems for black hole evolutions arXiv:gr-qc/0403034

[29] Leaver E W 1986 Spectral decomposition of the perturbation response of the Schwarzschild geometry Phys. Rev. D 34 384-408

[30] Pürrer M, Husa S and Aichelburg P C 2005 News from critical collapse: Bondi mass, tails and quasinormal modes Phys. Rev. D 71104005

[31] Zenginoğlu A 2008 A hyperboloidal study of tail decay rates for scalar and Yang-Mills fields Class. Quantum Grav. 25175013

[32] Teukolsky S A 1973 Perturbations of a rotating black hole: I. Fundamental equations for gravitational, electromagnetic, and neutrino-field perturbations Astrophys. J. 185 635-47

[33] Venter L R and Bishop N T 2006 Numerical validation of the Kerr metric in Bondi-Sachs form Phys. Rev. D 73084023

[34] Pretorius F and Israel W 1998 Quasi-spherical light cones of the Kerr geometry Class. Quantum Grav. 15 2289-301

[35] Friedrich H 1983 Cauchy problems for the conformal vacuum field equations in general relativity Commun. Math. Phys. 91 445-72

[36] Husa S, Schneemann C, Vogel T and Zenginoğlu A 2006 Hyperboloidal data and evolution AIP Conf. Proc. 841 306-13

[37] Andersson L 2002 Construction of hyperboloidal initial data Lect. Notes Phys. $604183-94$

[38] Newman E and Penrose R 1962 An approach to gravitational radiation by a method of spin coefficients J. Math Phys. 3 566-79

[39] Chandrasekhar S 1983 The Mathematical Theory of Black Holes (Oxford: Clarendon)

[40] Frolov V 1977 Newman-Penrose method in the general relativistic theory Sci. Acad. USSR 96 $72-180$ (in Russian)

[41] Ernst F J 1978 Coping with different languages in the null tetrad formulation of general relativity J. Math Phys. 19 489-93

[42] Newman E and Penrose R 1966 Note on the Bondi-Metzner-Sachs group J. Math Phys. 7 863-70

[43] Goldberg J N, Macfarlane A J, Newman E, Rohrlich F and Sudarshan E C G 1967 Spin s spherical harmonics and edth J. Math Phys. 8 2155-61

[44] Sarbach O and Tiglio M 2001 Gauge invariant perturbations of Schwarzschild black holes in horizon-penetrating coordinates Phys. Rev. D 64084016

[45] Brill D R, Cavallo J M and Isenberg J A 1980 K-surfaces in the Schwarzschild spacetime and the construction of lattice cosmologies J. Math. Phys. 21 2789-96

[46] Malec E and Murchadha N O 2003 Constant mean curvature slices in the extended Schwarzschild solution and collapse of the lapse: part I Phys. Rev. D 68124019

[47] Zenginoğlu A 2008 Hyperboloidal foliations and scri-fixing Class. Quantum Grav. 25145002

[48] Kreiss H O and Oliger J 1973 Methods for the Approximate Solution of Time Dependent Problems (Geneva: GARP Publication Series)

[49] Leaver E W 1985 An analytic representation for the quasi normal modes of Kerr black holes Proc. R. Soc. Lond. A 402 285-98

[50] Ching E S C, Leung P T, Suen W M and Young K 1995 Wave propagation in gravitational systems: late time behavior Phys. Rev. D 52 2118-32

[51] Price R 1972 Nonspherical perturbations of relativistic gravitational collapse: I. Scalar and gravitational perturtbations Phys. Rev. D 52419

[52] Gundlach C, Price R and Pullin J 1994 Late-time behaviour of stellar collapse and explosions: I. Linearized perturbations Phys. Rev. D 49 883-9 
[53] Gundlach C, Price R and Pullin J 1994 Late-time behaviour of stellar collapse and explosions: II. Nonlinear evolution Phys. Rev. D 49 890-9

[54] Barack L 1999 Late time dynamics of scalar perturbations outside black holes: I. A shell toy-model Phys. Rev. D 59044016

[55] Barack L 1999 Late time dynamics of scalar perturbations outside black holes: II. Schwarzschild geometry Phys. Rev. D 59044017

[56] Cunningham C T, Price R H and Moncrief V 1978 Radiation from collapsing relativistic stars: I. Linearized odd-parity radiation Astrophys. J. 224643

[57] Cunningham C T, Price R H and Moncrief V 1979 Radiation from collapsing relativistic stars: II. Linearized even-parity radiation Astrophys. J. 230 870-92

[58] Friedrich H and Stewart J 1983 Characteristic initial data and wavefront singularities in general relativity Proc. R. Soc. A 385 345-71

[59] Winicour J 2005 Characteristic evolution and matching Liv. Rev. Rel. 810

[60] Penrose R 1963 Asymptotic properties of fields and space-times Phys. Rev. Lett. 10 66-8

[61] Penrose R 1965 Zero rest-mass fields including gravitation: asymptotic behaviour Proc. R. Soc. Lond. A 284 159-203

[62] Moncrief V and Rinne O 2008 Regularity of the Einstein equations at future null infinity arXiv:0811.4109

[63] Zenginoğlu A 2008 Hyperboloidal evolution with the Einstein equations Class. Quantum Grav. 25195025 